\title{
Gravitational Riemann Invariants
}

\author{
Ph. Choquard \\ ITP.EPFL. $(\mathrm{CH})$ \\ Email: philippe.choquard@epfl.ch
}

\begin{abstract}
One-dimensional, inviscid, compressible and isentropic fluids under gravity are considered, here, as usefull preambles relevant to the theory of uni-axial meteorological phenomena, [4].We shaw that there are two new Riemann invariants, [1], incorporating gravity, which are constants of the motion. Expressing the mass densities occuring in these Invariants as product of their initial values times the inverse Jacobian of the characteristics of these fluids with respect to their initial values, we propose, central in this work, first order non-linear PDE's of Charpit type [2] satisfied by these invariants. Examples of solutions are given and checked to conform with results of, gravity-free, similar PDE's published in [3].
\end{abstract}

Keywords: 1-Dimensional, invicid, compressible, isentropic, gravity, Riemann Invriants

\section{Continuity and Euler Equatuins, Matrix Formulation, Invariants}

Let $z(y, t)$ be the coordinate of the characteristics at time $t$, with initial value $z(y, 0)=y>0$ and $z(y, t)<\infty$; let $g$ be the gravitational constant, $\rho(z, t) \geq 0$, the mass density, $u(z, t) \in \mathbb{R}$, the velocity field and $c_{S}(\rho)=$ const $\cdot \rho:=\varkappa \rho$, often and simply quoted as $c_{S}$, the isentropic sound velocity. With the

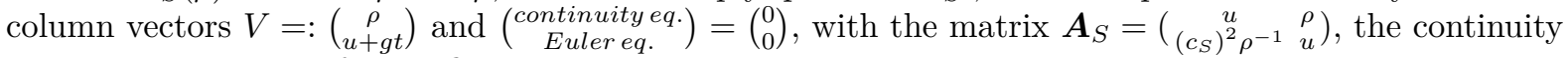
and Euler equations of these fluids are

$$
\frac{\partial}{\partial t}\left(\begin{array}{c}
\rho \\
u+g t
\end{array}\right)+\left(\begin{array}{cc}
u & \rho \\
c_{S}(\rho)^{2} \rho^{-1} & u
\end{array}\right) \frac{\partial}{\partial z}\left(\begin{array}{c}
\rho \\
u+g t
\end{array}\right)=\left(\begin{array}{cc}
\text { contuinity } & \text { eq. } \\
\text { Euler } & \text { eq. }
\end{array}\right)=\left(\begin{array}{l}
0 \\
0
\end{array}\right)
$$

and in a compact form

$$
\frac{\partial}{\partial t} V+\mathbf{A}_{S} \frac{\partial}{\partial z} V=\mathbf{0}
$$

The eigenvalues, $\lambda_{S}$, of the 2 by 2 matrix $\mathbf{A}_{S}$, are, in setting $\epsilon= \pm 1: \lambda_{S}=u+\epsilon \cdot c_{S}(\rho)$ and their eigenvectors are: $\left(\begin{array}{c}1 \\ \epsilon c_{S} \rho^{-1}\end{array}\right):=\vartheta_{\epsilon, S}$. A unique property of these eigenvectors, and., ipso facto, of the matrices $\boldsymbol{M}_{S}:=\left(\vartheta_{+1, S}, \vartheta_{-1, S}\right)$, is that they are constants since $c_{S} / \rho=\varkappa \neq 0$. With the diagonalizing vector $W$, i.e. $V=\boldsymbol{M}_{S} W$ and $A_{S} \boldsymbol{M}_{S}=\lambda_{S} \boldsymbol{M}_{S}, \boldsymbol{M}_{S} \neq \mathbf{0}$, the diagonalized version of the two PDE's (2) is

$$
\frac{\partial}{\partial t} W+\lambda_{S} \frac{\partial}{\partial z} W=0
$$

The indefinite integral solutions of (3) read

$$
\left.W(z, t ; \epsilon)=u+g t+\epsilon \int^{\rho(z, t)} d \rho \prime \cdot c_{S} \rho^{\prime}\right) \rho^{\prime-1}=u+g t+\epsilon c_{S}(\rho(z, t)) .
$$

It is readily checked that (4) are constants of the motion, properly identified as " Gravitationsl Riemann Invariants". We have indeed

$$
\frac{\partial}{\partial t} W+\lambda_{S} \frac{\partial}{\partial z} W=(\text { Euler eq. })+\left(\epsilon c_{S} / \rho\right) \cdot(\text { continuity eq. })=0 .
$$

Lastly, if $y$.is the initial value of $z, W(y, 0 ; \epsilon)$, that of $W(z, t ; \epsilon)$, we have

$$
W(z, t ; \epsilon)=W(y, 0 ; \epsilon)
$$




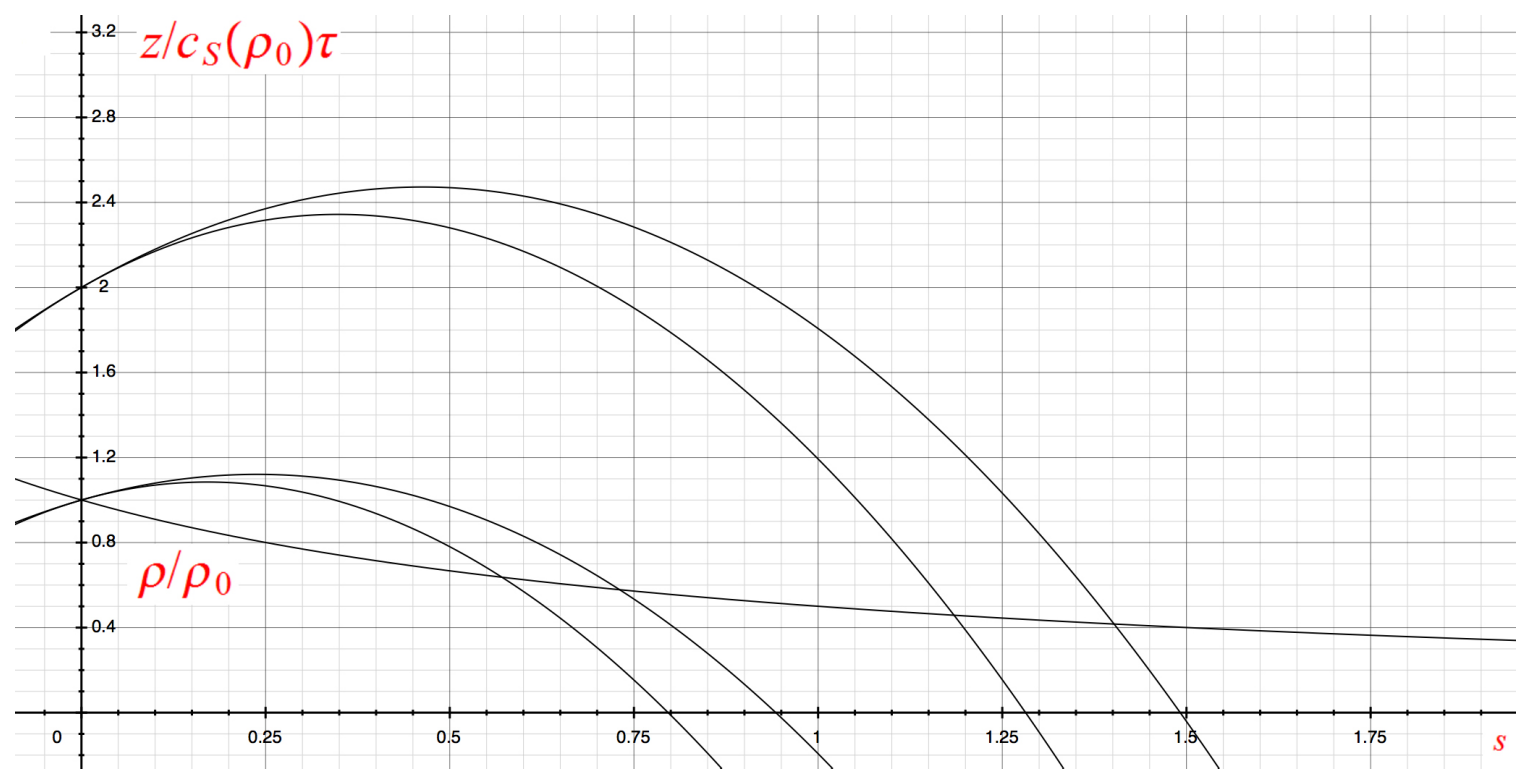

Figure 1. Chaeacteristics (9) and Density (10)

\section{PDEs of (6)}

Consider next the formal solution of the densities occuring in [6]. Since $\rho(z, t) d z=\rho_{0}(y) d y$ and with the Jacobian $\partial z(y, t) / \partial y$, we find

$$
\rho(z, t)=\rho_{0}(y)(\partial z / \partial y)^{-1}
$$

Central in this work, we obtain the following first order, non-linear PDE's of Charpit type, for the two isentropic cases given by [6], and with $u(z, t)=\partial z / \partial t$, namely

$$
\partial z / \partial t+\epsilon c_{S}\left(\rho_{0}(y)\right)\left((\partial z / \partial y)^{-1}-1\right)+g t-u(y)=0
$$

\section{Examples}

Using the Charpit scheme, whose general purpose is to convert first order non-linear PDE's of, say $2 n$ independent variables in a set of $2 n$ ODE's [2] and to solve the latter, a purpose similar but more general than that of Hamilton-Jacobi's scheme in Analytical Mechanics, our examples consist of systems of two independant variables, i.e. $y$ and $t$. Considering, for illustration, the case $\rho_{0}(y)=\rho_{0}$ and $u(y)=y / \tau, \tau$ being a reference time constant, we find, in setting $g=0$, two particular solutions of the general ones given in $[3]$

$$
z(y, t)=(1+t / \tau)\left(y+\epsilon c_{S}\left(\rho_{0}\right) \tau\right)-\epsilon c_{S}\left(\rho_{0}\right) \tau-\frac{1}{2} g t^{2}-\epsilon c_{S}\left(\rho_{0}\right) \ln (1+t / \tau)
$$

and the equation for the density is simply:

$$
\rho(y, t)=\rho_{0}(1+t / \tau)^{-1} .
$$

On Fig. 1, four examples of the characteristics [9] are presented and one for the density [10] the latter, plotted in units of $\rho_{0}$. If $s=t / \tau$, if $z$ and if $y$ are plotted in units $c_{S}\left(\rho_{0}\right) \tau$ while $g$, in units of $c_{S}\left(\rho_{0}\right) / \tau$, is choosen to be $=5$, then, the four figures shown correspond to the initial values $(\epsilon, y)=$ $((-1,1),(1,1),(-1,2),(1,2))$ : 
Acknowledgments. The author is very much indebted to J. Stubbe of EPFL for his sustained interest and usefull suggestions in the course of this work, to J. Gurtner of ETML to A. Ramella and E.Batori, architecture and physics students at EPFL, for their precious help in the preparation of the figures and of the poster, presented at the IUPAP Statphys.26 meeting, held in Lyon (Fr), 7,18-22,.2016.

\section{References}

1. B. Riemann, Abhandlung der königlichen Gesellschaft der Wissenschaften zu Göttingen, Vol. 8, 1860

2. L. Deborath, Nonlinear PDE's for Scientists and Engineers 3th. edition ch.4 sect,4.2 Birkhauser, 2011

3. A.D. Polyanin, V.F. Zaitzev, Handbook of Nonlinear PDE's (2th. edition), ch.2 sect.2.1.2, No 3,p.45, Taylor and Francis, 2012.

4. Ph. Choquard, M. Vuffray, Physica D, vol. 285, X 2014, pp. 11-22,sect.4, items 3 \& 4 